\title{
Dysmorphic Photoreceptors in a P23H Mutant Rhodopsin Model of Retinitis Pigmentosa Are Metabolically Active and Capable of Regenerating to Reverse Retinal Degeneration
}

\author{
Damian C. Lee, ${ }^{1}$ Felix R. Vazquez-Chona, ${ }^{2}$ W. Drew Ferrell, ${ }^{2}$ Beatrice M. Tam, ${ }^{1}$ Bryan W. Jones, ${ }^{2}$ Robert E. Marc, ${ }^{2}$ \\ and Orson L. Moritz ${ }^{1}$ \\ ${ }^{1}$ Department of Ophthalmology and Visual Sciences and Centre for Macular Research, University of British Columbia, Vancouver, British Columbia, \\ Canada, V5Z 3N9, and 2Department of Ophthalmology, John A. Moran Eye Center, University of Utah, Salt Lake City, Utah 84132
}

This study evaluated the capacity of Xenopus laevis retina to regenerate photoreceptor cells after cyclic light-mediated acute rod photoreceptor degeneration in a transgenic $\mathrm{P} 23 \mathrm{H}$ mutant rhodopsin model of retinits pigmentosa. After discontinuation of cyclic light exposure, we monitored histologic progression of retinal regeneration over a 3 week recovery period. To assess their metabolomic states, contralateral eyes were processed for computational molecular phenotyping. We found that retinal degeneration in the $\mathrm{P} 23 \mathrm{H}$ rhodopsin mutation could be partially reversed, with regeneration of rod photoreceptors recovering normal morphology (including full-length rod outer segments) by the end of the 3 week recovery period. In contrast, retinal degeneration mediated by directly induced apoptosis did not recover in the 3 week recovery period. Dystrophic rod photoreceptors with truncated rod outer segments were identified as the likely source of rod photoreceptor regeneration in the $\mathrm{P} 23 \mathrm{H}$ retinas. These dystrophic photoreceptors remain metabolically active despite having lost most of their outer segments.

\section{Introduction}

Retinitis pigmentosa (RP) is a hereditary degenerative disease of the retina that causes progressive loss of rod and cone photoreceptors. In some forms of RP, rod cell death is followed by secondary cone photoreceptor dysfunction and death. Clinically, symptoms in this form manifest as night blindness and tunnel vision, followed by progressive loss of color and central vision (Milam et al., 1998). RP is genetically heterogeneous, with mutations in $>30$ distinct genes linked to the disease and $>100$ gene defects in rhodopsin alone (Berson, 1993; Hartong et al., 2006). Despite this genetic heterogeneity, the most common initial and primary result of the disease is thought to be dysfunction and death of rod photoreceptors.

There is no known cure for RP. Current approaches to development of therapies have focused on attenuating symptoms (for

\footnotetext{
Received Sept. 13, 2011; revised Dec. 15, 2011; accepted Dec. 23, 2011.

Author contributions: D.C.L., F.R.V.-C., R.E.M., and 0.L.M. designed research; D.C.L., F.R.V.-C., and W.D.F. performed research; D.C.L., F.R.V.-C., W.D.F., B.M.T., B.W.J., and 0.L.M. analyzed data; D.C.L., F.R.V.-C., W.D.F., B.M.T., B.W.J., R.E.M., and O.L.M. wrote the paper.

This work was supported by Foundation Fighting Blindness-Canada and Canadian Institutes of Health Research grants (0.L.M.), National Institutes of Health Grants EY02576, EY015128, and EY014800 Vision Core (R.E.M.), Research to Prevent Blindness Career Development Award and Edward N. and Della L. Thome Memorial Foundation grants (B.W.J.), Fight for Sight (F.V.C., W.D.F.), International Retinal Research Foundation, Knights Templar Eye Foundation, and National Institutes of Health Developmental Biology Training Grant Fellowship 5T32 HD07491 (F.V.C.), and University of Utah Undergraduate Research Opportunities Program (W.D.F.).

R.E.M. is Chief Executive Officer of Signature Immunologics (Salt Lake City, UT).

Correspondence should be addressed to Orson L. Moritz, Department of Ophthalmology and Visual Sciences and Centre for Macular Research, University of British Columbia/Vancouver General Hospital Eye Care Centre, 2550 Willow Street, Vancouver, BC, Canada, V5Z3N9. E-mail: olmoritz@mail.ubc.ca.

DOI:10.1523/JNEUROSCI.4752-11.2012

Copyright $\odot 2012$ the authors $\quad 0270-6474 / 12 / 322121-08 \$ 15.00 / 0$
}

example, by avoiding light exposure or vitamin A supplementation), gene therapy, restoration of lost photoreceptors by stem cell transplantation, and retinal prostheses (Shintani et al., 2009). An alternative strategy would be to stimulate endogenous survivor cells to regrow and repair damaged or lost photoreceptors, reversing retinal degeneration. This therapeutic approach avoids immunologic complications from donor incompatibility and implant rejection and side effects of immunosuppressant drugs.

We have previously developed and characterized a transgenic Xenopus laevis bovine $\mathrm{P} 23 \mathrm{H}$ rhodopsin $(\mathrm{bP} 23 \mathrm{H})$ model of inducible rod degeneration based on the human proline-to-histidine substitution at position $23(\mathrm{P} 23 \mathrm{H})$ of rhodopsin (Tam and Moritz, 2007). The $\mathrm{P} 23 \mathrm{H}$ rhodopsin mutation is responsible for the majority of cases of autosomal-dominant RP (adRP) in North America (Dryja et al., 1990; Sohocki et al., 2001). Previous studies have suggested that the mutant $\mathrm{P} 23 \mathrm{H}$ protein is misfolded and retained in the endoplasmic reticulum (ER) (Kaushal and Khorana, 1994; Saliba et al., 2002; Noorwez et al., 2003; Noorwez et al., 2004; Tam and Moritz, 2006). As observed in other protein misfolding disorders, prolonged ER stress attributable to the accumulation of mutant protein ultimately leads to rod photoreceptor cell death (Lin et al., 2008). Interestingly, we found previously that, in our transgenic $X$. laevis bP23H model, dark rearing prevented rod degeneration in the $14 \mathrm{~d}$ post-fertilization (dpf) animals examined (Tam and Moritz, 2007).

In this study, we tested whether the X. laevis retina is capable of regenerating after targeted acute cellular damage, specifically, rod photoreceptor degeneration in this model of RP. We also determined the long-term effects and retinal responses of this inducible model of RP after acute induction of a retinal degener- 
ation signal. Our results show that $X$. laevis tadpoles are able to reverse $\mathrm{bP} 23 \mathrm{H}$ mediated degeneration and regenerate lost rod photoreceptor outer segments after the degeneration stimulus (cyclic light) is removed. We also show that degenerating bP23H photoreceptors are metabolically active despite having completely lost their outer segments.

\section{Materials and Methods}

Transgenic $\mathrm{X}$. laevis generation and rearing. We have previously developed and extensively characterized transgenic $X$. laevis inducible models of $\mathrm{RP}$, including light-inducible bP23H (Tam and Moritz, 2007) and drug-inducible caspase 9 (iCasp9) models (Hamm et al., 2009). Transgenic $X$. laevis tadpoles expressing either $\mathrm{bP} 23 \mathrm{H}$ or iCasp9 in the rod photoreceptors were bred from transgenic frogs carrying the respective transgenes under the control of the $X$. laevis opsin promoter. Green fluorescent protein (GFP)-labeled rod photoreceptors were generated by mating with transgenic frogs expressing eGFP under the X. laevis opsin promoter (Tam et al., 2000). Tadpoles were reared in an $18^{\circ} \mathrm{C}$ incubator on a $12 \mathrm{~h}$ dark/light (1700 lux) cycle, unless otherwise specified. Rod photoreceptor death was induced in the iCasp9 tadpoles by the addition of $10 \mathrm{~nm}$ AP20187 (Ariad Pharmaceuticals) to the tadpole rearing medium as described previously (Hamm et al., 2009).

Immunohistochemistry and confocal microscopy. The preparation of tadpole retinas for immunohistochemistry was performed as described previously (Tam and Moritz, 2007). Tadpoles were killed, and the eyes were enucleated and fixed overnight with $4 \%$ formaldehyde in $0.1 \mathrm{M}$ sodium phosphate buffer, $\mathrm{pH}$ 7.4. The fixed eyes were dissected and cryoprotected by infiltration with $20 \%$ sucrose solution in $0.1 \mathrm{M}$ sodium phosphate buffer, $\mathrm{pH}$ 7.4. The cryoprotected eyes were embedded in Tissue-Tek O.C.T. compound (Sakura Finetek) and frozen. Serial cryosections, $12 \mu \mathrm{m}$, were cut and collected on Fisherbrand Superfrost/Plus microslides (Thermo Fisher Scientific). Retinal sections were labeled with wheat germ agglutinin (WGA) conjugated to Alexa Fluor 488 or 555, diluted 1:200 (Invitrogen), and Hoescht 33342, diluted 1:1000 (Sigma-Aldrich), to label rod outer segments (ROS) and nuclei, respectively. TUNEL staining was performed with the ApopTag Red in situ apoptosis detection kit as per the recommendations of the manufacturer (Millipore). Confocal images were acquired using Zeiss 510 Meta laser scanning confocal microscope (Carl Zeiss). Only representative images were shown.

Metabolic labeling and electron microscopy. Enucleated eyes for metabolic labeling were fixed in $2.5 \%$ glutaraldehyde/ $1 \%$ formaldehyde in $0.1 \mathrm{M}$ cacodylate buffer with $3 \%$ sucrose, $\mathrm{pH} 7.4$, for a minimum of $1 \mathrm{~d}$. The fixed eyes were subsequently stained en bloc in $1 \%$ osmium tetroxide in $0.1 \mathrm{M}$ cacodylate buffer, $\mathrm{pH} 7.4$, for $1 \mathrm{~h}$, followed by $1 \%$ uranyl acetate in maleate buffer for $1 \mathrm{~h}$, and dehydrated in a graded methanol/acetone series (50-100\%). The dehydrated eyes were infiltrated and embedded in EMbed 812 epoxy resin (Electron Microscopy Sciences). Retinas were serially sectioned at $90 \mathrm{~nm}$ onto 12-well HTC Cel-Line slides (Erie Scientific) and probed with antihapten IgGs (Marc et al., 1995, 1998a,b, 2007; Marc and Jones, 2002) (http:// prometheus.med.utah.edu/ $\sim$ marclab/protocols.html). Anti-hapten IgGs from Signature Immunologics (L-aspartate, L-glutamate, glycine, glutathione, GABA, arginine, alanine, and taurine) and from J. C. Saari (University of Washington, Seattle, WA) [cellular retinaldehyde-binding protein (CRALBP)] have been characterized extensively in previous publications
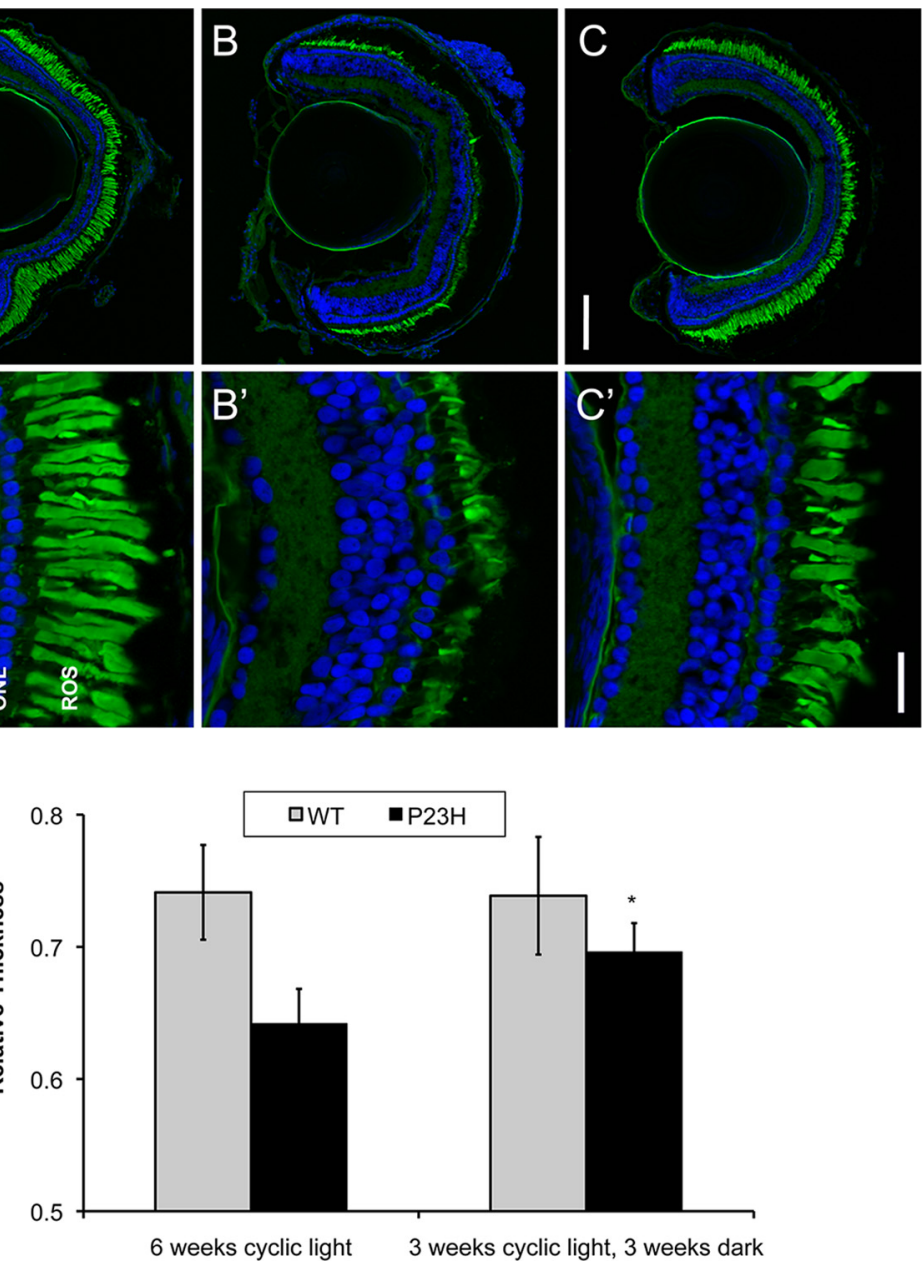

Figure 1. Partial repopulation of the retina with rod photoreceptors in bP23H animals. $A, A^{\prime}$, Wild-type retina. $B, B^{\prime}, b P 23 H$ transgenic retina reared in cyclic light since fertilization. $C^{\prime} C^{\prime}$, P23H transgenic retina reared in complete darkness for 3 weeks afte inger lexiform layer; GCL, ganglion cell layer. Scale bars: top, $100 \mu \mathrm{m}$; bottom, $20 \mu \mathrm{m}$. D, Relative 列 $(n>6$, mean \pm SD). The asterisk indicates that the relative thickness of the dark-recovered bP23H retina is significantly thicker than the cyclic light-reared bP23H retina (Student's $t$ test, $p<0.0002$ ). WT, Wild-type.

(Marc et al., 1995, 1998a,b, 2007; Marc and Jones, 2002). IgG binding was visualized with silver intensification of $1.4 \mathrm{~nm}$ gold granules coupled to species-specific secondary IgGs (Nanoprobes), captured as 8-bit monochrome images at a resolution of $244 \mathrm{~nm} /$ pixel, mosaiced into arrays, and registered using ir-tweak software (http://www.sci.utah.edu/download/ ncrtoolset). Monochrome images were density mapped, and red-greenblue images were intensity mapped (Marc et al., 1995, 1998a,b, 2007; Marc and Jones, 2002). All images were prepared with Adobe Photoshop CS3 (Adobe Systems). Cell classification (K-means clustering and histogram analysis) was performed as described previously on monochrome images using pixel-based approaches (Marc et al., 1995; Marc and Jones, 2002). Data clusters were visualized as theme maps [CMP (computational molecular phenotyping); a clustering package created at the Scientific Computing Institute, University of Utah], and Adobe Photoshop CS3 (Adobe Systems) was used for final image generation.

All animal procedures were performed in accordance with the ARVO Statement for the Use of Animals in Ophthalmic and Vision Research.

\section{Results}

Dysmorphic transgenic bP23H rod photoreceptors are able to regenerate outer segments

The bP23H model of retinal degeneration was developed previously and characterized by Tam et al. (2007). bP23H transgenic 
retinas experience light-dependent degeneration of rods in which ROS are essentially ablated, and total rhodopsin levels are significantly reduced in light-exposed $\mathrm{bP} 23 \mathrm{H}$ retinas. We previously found that dark rearing prevents this degeneration in animals up to $14 \mathrm{dpf}$, restoring total rhodopsin levels to near wild-type levels (Tam and Moritz, 2007). To evaluate the ability of bP23H retinas to regenerate when the degeneration signal was removed (i.e., acute light exposure), bP23H transgenic tadpoles were reared in cyclic light condition for $21 \mathrm{~d}$ to induce rod degeneration and subsequently moved to complete darkness for 3 weeks. After the 3 week recovery period, tadpoles were killed and fixed in $4 \%$ formaldehyde. Retinal cryosections were prepared for immunohistochemistry and examined by laser scanning confocal microscopy. We found that the ROS layer of the dark-recovered animals (Fig. 1C, $C^{\prime}$ ) was thicker than that of cyclic light-reared animals (Fig. $1 B, B^{\prime}$ ) and comparable with wild-type controls (Fig. $\left.1 A, A^{\prime}\right)$, i.e., an increase in retinal thickness occurred in darkrecovered transgenic eyes versus cyclic light-reared eyes (Fig. $1 D$ ). These observations indicate that $\mathrm{bP} 23 \mathrm{H}$ ROS regenerate when the degeneration stimulus is removed by dark rearing.

\section{Rod photoreceptors survive in $\mathrm{bP} 23 \mathrm{H}$ degenerated retinas}

To identify the source of the regenerating bP23H ROS, we generated transgenic tadpoles that expressed both the degenerationinducing bP23H transgene and GFP in rods. GFP was expressed in the rod photoreceptors under the control of the rod-specific Xenopus rod opsin promoter (Tam et al., 2000), effectively labeling rod photoreceptors in vivo (Fig. $2 \mathrm{~A}$ ). The GFP/bP23H transgenic tadpoles were reared in cyclic light for 2 weeks to induce rod degeneration. For comparison, we induced apoptosis in doubletransgenic GFP/iCasp9 tadpoles (Fig. 2 B) (Hamm et al., 2009). These animals express a modified form of caspase 9 (iCasp9) in their rod photoreceptors, which can be activated by application of the drug AP20187, causing apoptotic cell death. In all the animals we examined, degenerating $\mathrm{bP} 23 \mathrm{H}$ retinas retained GFPpositive inner segments of rod photoreceptors that had severely truncated outer segments (Fig. 2C). In contrast, we found that direct induction of apoptosis in iCasp9 retinas (see Materials and Methods) resulted in no detectable GFP signal in the photoreceptor layer [outer nuclear layer (ONL), inner segment and outer segment] (Fig. 2D), indicating the absence of rod photoreceptors. This was verified by the absence of rhodopsin immunoreactivity in the same region (data not shown). These observations suggest that numerous $\mathrm{bP} 23 \mathrm{H}$ rod photoreceptors continue to persist in the photoreceptor layer in a morphologically abnormal form, whereas rod photoreceptors that undergo apoptosis are rapidly lost and cleared from the retina.

We continued to monitor the GFP-labeled rods of bP23H tadpoles reared in cyclic light over a period of 6 weeks. We found that the degenerating $\mathrm{bP} 23 \mathrm{H}$ rod cell bodies persisted in the ONL of the retina for up to 6 weeks in cyclic light (Fig. $3 A$ ). We also observed that the remaining number of GFP-labeled rods decreased gradually over time in cyclic light (Fig. $3 B$ ), suggesting a constant, low rate of rod photoreceptors loss. However, the morphologically abnormal rods that remained after 6 weeks in cyclic light retained the ability to recover their normal morphology and restore the ROS layer (Fig. 3C) after the degeneration stimulus was removed by subsequent dark rearing.

Because of our finding that rods persisted in degenerated $\mathrm{bP} 23 \mathrm{H}$ retinas, we wanted to measure the extent of cell death in cyclic light-exposed bP23H retinas of older tadpoles. bP23H and wild-type tadpoles were raised in normal rearing conditions (cyclic light) for 9 weeks. Histological sections of these retinas over
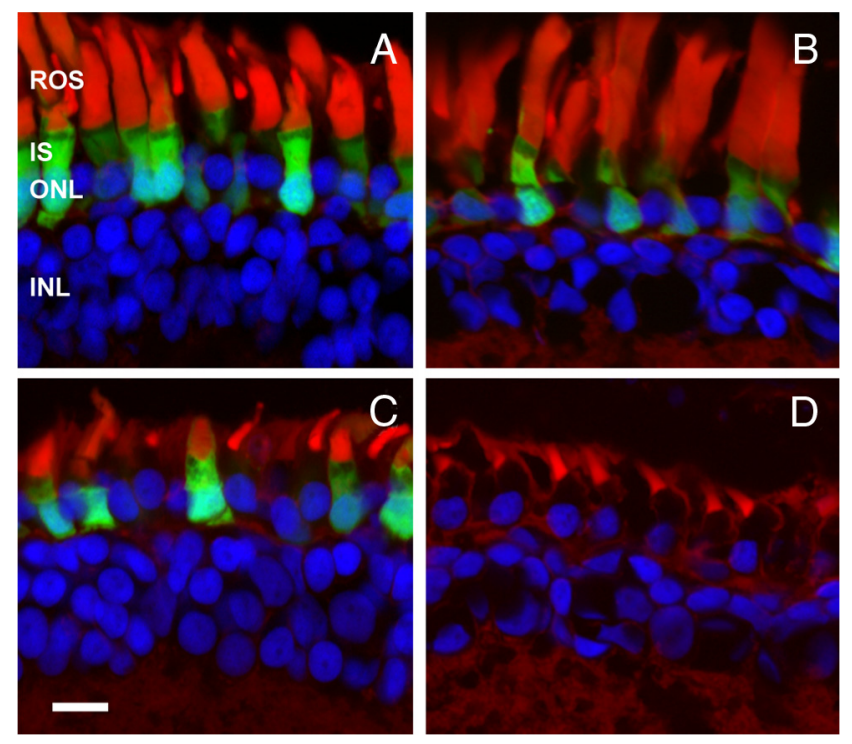

Figure 2. Dysmorphic bP23H rod photoreceptors with truncated outer segments identified via GFP fluorescence. Representative confocal micrographs of two different models of retinal degeneration before (top) and after (bottom) degeneration. $\boldsymbol{A}, \boldsymbol{C}$, bP23H/GFP doubletransgenic retinas. GFP was coexpressed in the rods of bP23H tadpoles to label rod photoreceptors. $\boldsymbol{A}$, Dark-reared bP23H/GFP retina. $\boldsymbol{C}$, Degenerating bP23H retina after 6 weeks in cyclic light. Note the presence of GFP-positive rod cell bodies with severely truncated outer segments. $\boldsymbol{B}, \boldsymbol{D}$, iCasp9/GFP double-transgenic retinas. $\boldsymbol{B}$, iCasp9/GFP retina before induction of apoptosis. D, iCasp9/GFP retina $4 \mathrm{~d}$ after administrating $10 \mathrm{~mm}$ AP20187. Rod photoreceptors are permanently lost and noticeably absent after drug-induced apoptosis. Sections were stained with Alexa Fluor 555-conjugated WGA to visualize membranes (red) merged with Hoechst nuclear stain (blue). Rod photoreceptors were visualized by GFP expression (green). IS, Inner segment; INL, inner nuclear layer. Scale bar, $10 \mu \mathrm{m}$.

the 9 week period in cyclic light were prepared as described above. Apoptotic cells were identified by TUNEL assay in bP23H and wild-type retinas, and the number of TUNEL-positive cells in the ONL were scored (Fig. 4). We found that there was a low level of apoptosis in $\mathrm{bP} 23 \mathrm{H}$ degenerating retinas, which was not significantly different from that observed in wild-type retinas in cyclic light.

To identify the acute effects of cyclic light on bP23H rods, we determined the time course of cell death immediately after light exposure. Transgenic bP23H and wild-type tadpoles were initially raised in complete darkness and moved to cyclic light at 14 $\mathrm{dpf}$ (12 h light/dark cycle). At 2, 5, 13, and $19 \mathrm{~d}$ in cyclic light, retinas were analyzed by histology and TUNEL. We observed that, after two cycles of light exposure, there was an increase in TUNEL-positive ONL cells in $\mathrm{bP} 23 \mathrm{H}$ retinas, which was not observed in wild-type retinas (Fig. $5 A, B$ ). By 13 cycles of light, there was decrease in the level of apoptosis, approaching levels comparable with wild-type retinas in cyclic light. Together, the data suggest that, on light exposure, there is an initial wave of cell death involving a subset of rod photoreceptors. The remaining rods degenerate but die relatively slowly, retaining the capacity to regenerate their OS when light is removed.

\section{Metabolomic status of degenerating and recovering bP23H retinas}

To investigate the metabolic properties of $\mathrm{P} 23 \mathrm{H}$ photoreceptors, we visualized small-molecule distributions in the retina using CMP (Fig. 6) (Marc et al., 1995, 1998a,b, 2007; Marc and Jones, 2002). CMP measures metabolite concentration as $\mathrm{N}$-dimensional molecular signals with anti-hapten antibodies. Of interest was the distribution of metabolites involved in photoreceptor osmoregulation 
A

$\mathrm{P} 23 \mathrm{H}$

WT
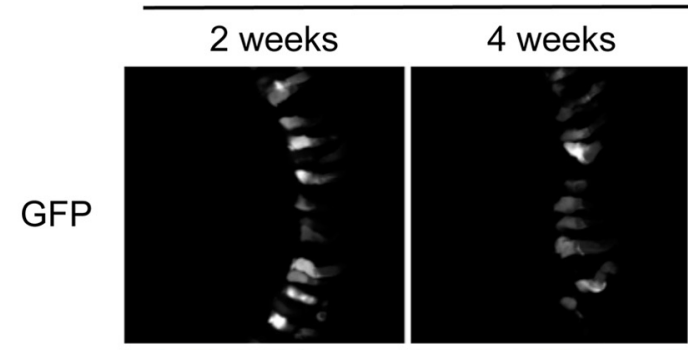

\section{6 weeks}
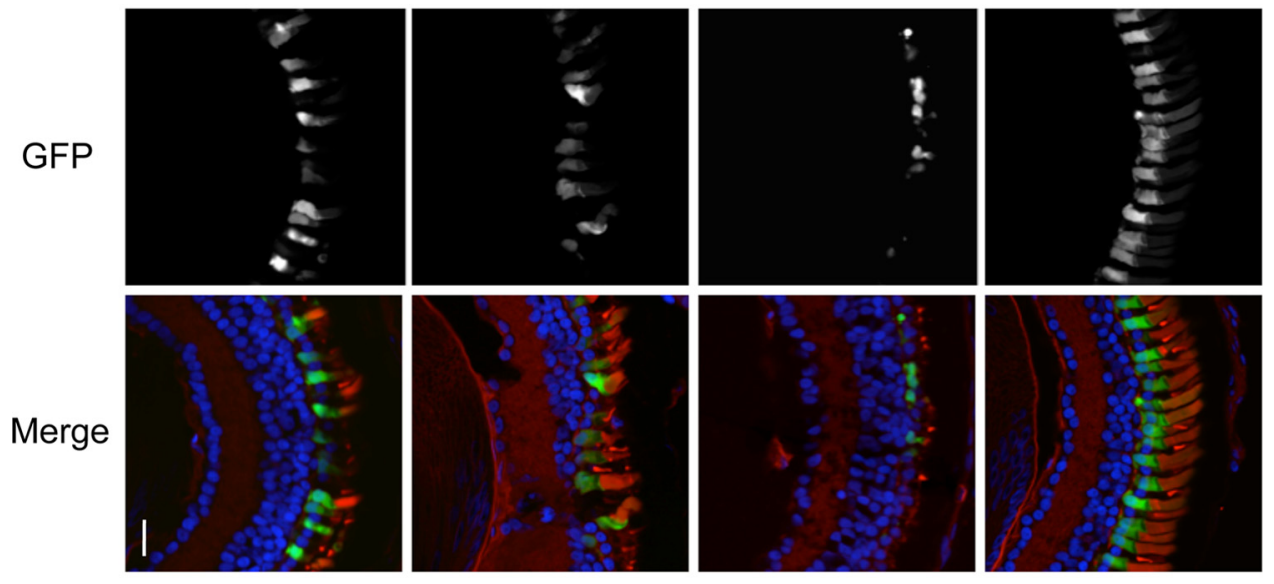

B
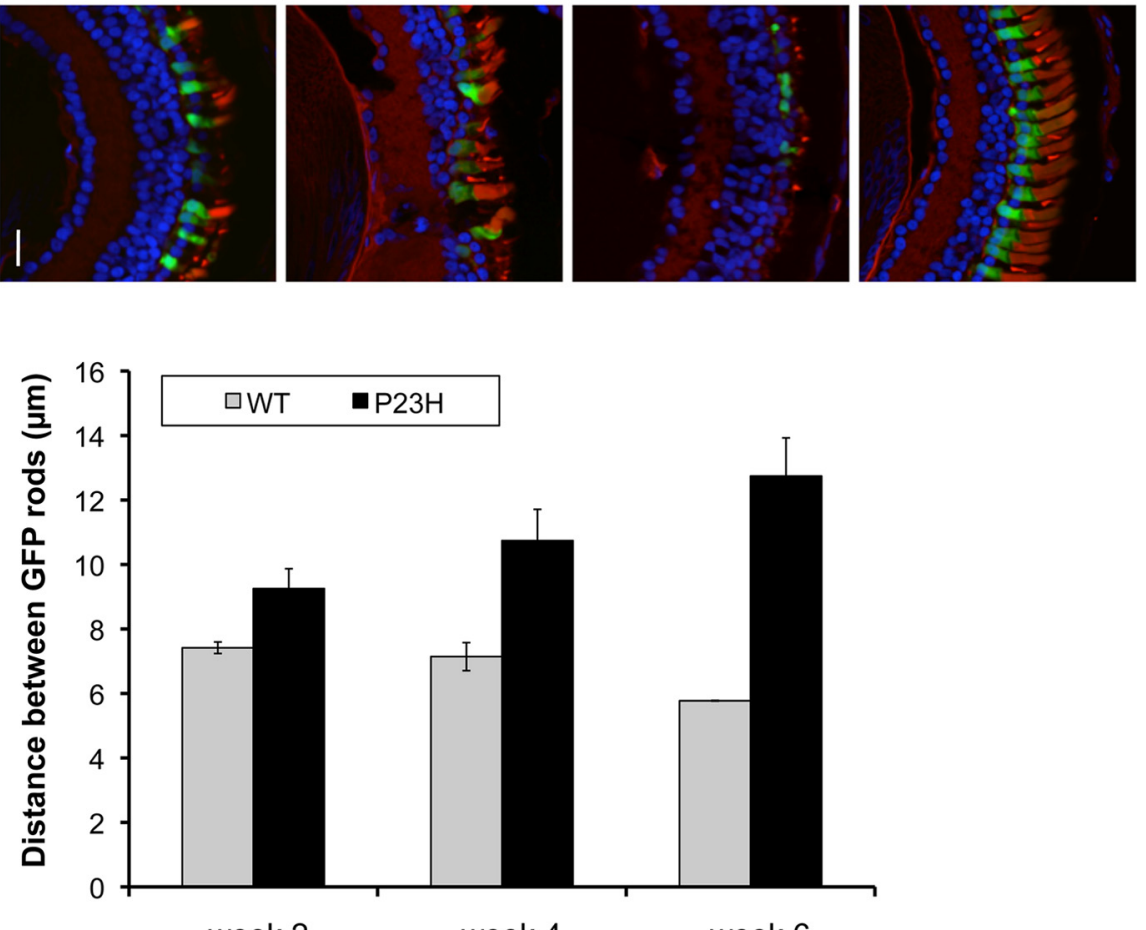

Time in cyclic light

C

WT

$\mathrm{P} 23 \mathrm{H}$

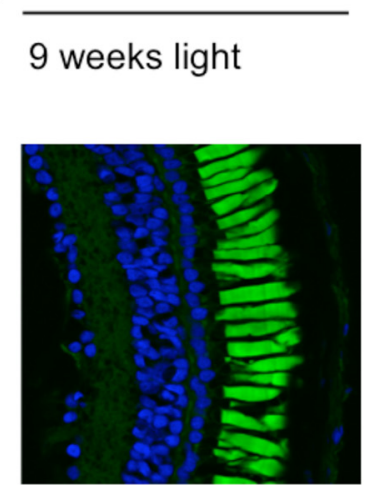

6 weeks light

6 weeks light, 3 weeks dark
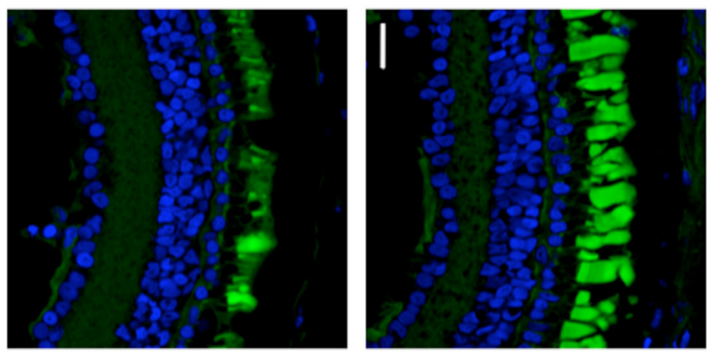

Figure 3. Dysmorphic rhoP23H rod cells are able to regenerate in the dark. $A$, Dysmorphic rod cells can be observed in the degenerating bP23H retina for up to 6 weeks in cyclic light-rearing conditions. Sections were stained with Alexa Fluor 555-conjugated WGA to visualize membranes (red) merged with Hoechst nuclear stain (blue). Rod photoreceptors were visualized by GFP expression (green). B, Relative density of GFP-positive rods in the central retina over time ( $n=3$, mean \pm SEM). C, bP23H rod cells are able to regenerate outer segments lost after 6 weeks in cyclic light. Cessation of degenerating light signal reverses ROS degeneration. Sections were stained with WGA to visualize membranes (green) merged with Hoechst nuclear stain (blue). Scale bars, $20 \mu \mathrm{m}$. WT, Wild type.

(taurine, Fig. $6 A-C$ ) and core metabolism (glutamate, Fig. 6D-F), as well as in metabolites involved in redox function (glutathione, Fig. $6 G-I)$.

In control wild-type retina, the photoreceptor cell body, inner segments, and the ellipsoid displayed abundant levels of taurine
(Fig. 6A) and glutamate (Fig. 6D) but low levels of glutathione (Fig. $6 G$ ). Assigning the small-molecule signals taurine, glutathione, and glutamate to red, green, or blue channels, respectively (Fig. 6J), displays small-molecule profiles of photoreceptors (pink-red) retinal pigment epithelium (RPE) (green, as a result 


\section{9 weeks cyclic light}

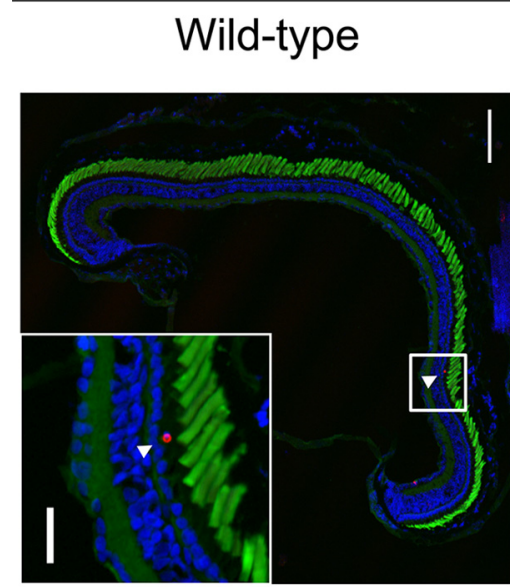

\section{$\mathrm{bP} 23 \mathrm{H}$}

TUNEL positive ONL cells

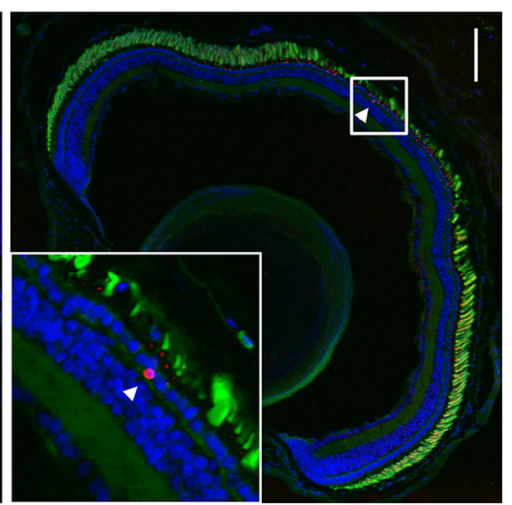

$0.6 \pm 0.2$

Figure 4. Apoptosis in bP23H retinas reared in cyclic light is comparable with WT retinas in cyclic light. Comparison of wild-type retina (left) versus bP23H retina (right) after 9 weeks in cyclic light. The arrowheads point to TUNEL-positive (red) 0NL cells. Insets show enlargements of respective boxed areas. The number of TUNEL-positive cells in the ONL were counted over a 9 week period. The highest number of TUNEL-positive ONL cells per section per retina at week 9 is shown ( $n>3$, mean \pm SEM). WGA, Green; Hoechst nuclear stain, blue. Scale bars: $100 \mu \mathrm{m}$; inset, $10 \mu \mathrm{m}$.

of high glutathione content) or ganglion cell profiles (blue, as a result of the high glutamate content and low taurine content). Clustering of multispectral $N$-dimensional data with additional small-molecule signals (aspartate, GABA, glycine, arginine, alanine, and CRALBP) shown as a theme map demonstrated that rods and cones had similar metabolomic profiles (Fig. $6 \mathrm{M}$, green channel). Cones and rods showed similar content for glycine and aspartate, with low or no levels of GABA, arginine, alanine, and CRALBP.

In degenerating $\mathrm{bP} 23 \mathrm{H}$ retinas, surviving cones and rods maintained high levels of taurine and glutamate (Fig. $6 B, E$ ) despite having disorganized outer segments. In areas in which the rod cells were ablated (Fig. 6B,E, arrows), CRALBP-positive Müller glia filled the void. Surprisingly, taurine-glutathioneglutamate metabolic envelopes (Fig. $6 \mathrm{~K}$ ) and clustering of multispectral $N$-dimensional data (Fig. $6 N$, green channel) in degenerating retinas showed similar metabolomic profiles between affected rods and unaffected cones.

In recovering $\mathrm{bP} 23 \mathrm{H}$ retinas, rods exhibited decreased taurine levels with enhanced glutamate and glutathione content (Fig. $6 C, F, I$, arrowheads). Metabolic maps (Fig. $6 L$ ) and theme maps (Fig. 6O) of recovering bP23H retina demonstrate that cone populations (Fig. 6O, green channel) exhibit stable metabolomic profiles, whereas rods display variability in taurine, glutamate, and glutathione content (Fig. 60, yellow, blue, and magenta channels). Interestingly, recovering rods with enhanced glutamate and glutathione content also had elongated somas similar to those found in the normal retina.

\section{Discussion}

In this study, we demonstrate that light-induced retinal degeneration associated with the $\mathrm{bP} 23 \mathrm{H}$ rhodopsin mutant is partially reversible. After an initial surge of cell death, dystrophic but viable rod photoreceptors persist in the retina for considerable time, with a low rate of cell death. These rods are metabolically active

and able to regrow lost outer segments if light is removed, partially restoring the ROS layer.

The $\mathrm{P} 23 \mathrm{H}$ mutant rhodopsin form of adRP has been studied extensively in animal models. Jones et al. (2003) showed that the transgenic GHL (V20G, P23H, $\mathrm{P} 27 \mathrm{~L}$ ) mouse and transgenic $\mathrm{P} 23 \mathrm{H}$ rat undergo an early phase of photoreceptor stress followed by a later phase of photoreceptor death. Although the precise molecular mechanisms leading to rod cell death are not completely clear, the $\mathrm{P} 23 \mathrm{H}$ rhodopsin mutant protein has been found to form aggregates in the ER of cells in culture and rod photoreceptors of Drosophila and $X$. laevis retina (Kaushal and Khorana, 1994; Galy et al., 2005; Tam and Moritz, 2006, 2007; Tam et al., 2010). In our $X$. laevis bP23H model, this aggregation is exacerbated by light exposure and is modulated by vitamin A (Tam and Moritz, 2006, 2007; Tam et al., 2010).

Proteins in the secretory pathway can be retained in the ER if they are unable to fold properly. ER retention of misfolded proteins induces the unfolded protein response (UPR) pathway, with a primary role to relieve ER stress. Stress is reduced via increased degradation of misfolded proteins, upregulation of chaperones to improve protein folding, and global reduction of protein translation. If the UPR is unable to recover, continued ER stress responses induce programmed cell death pathways to eliminate the stressed cells (Lin et al., 2008; Wang et al., 2010). This is a potentially useful response to such insults as viral infection.

Our working hypothesis is that the misfolding of $\mathrm{P} 23 \mathrm{H}$ rhodopsin is light dependent because the mutant protein is stabilized by binding to the unbleached chromophore 11-cis-retinal (Moritz and Tam, 2010; Tam et al., 2010). Under light conditions, $\mathrm{P} 23 \mathrm{H}$ is unable to fold before ER exit, likely leading to ER stress and consequent ER stress responses, such as the UPR as cytoprotective mechanisms. Our study indicates that there is an initial wave of cell death but that the majority of rod cells survive this period, likely because of cytoprotective mechanisms. One cytoprotective consequence of ER stress is reduced protein synthesis, which is the likely cause of ROS regression in our model. Once the degeneration-inducing stimulus (light) is removed, the increased supply of 11-cis-retinal stabilizes $\mathrm{P} 23 \mathrm{H}$ rhodopsin and normal cellular function is restored, allowing ROS to regrow. It has been reported previously that, in $\mathrm{P} 23 \mathrm{H}-3$ rats, low light reverses rod degeneration (Jozwick et al., 2006; Valter et al., 2008), and the overexpression of the chaperone BiP restores visual function (Gorbatyuk et al., 2010), suggesting that similar processes may occur in other P23H-rhodopsin-based models of retinal degeneration. Recently, Tam et al. (2010) showed that vitamin A modulates the trafficking of the $\mathrm{bP} 23 \mathrm{H}$ protein, acting as a pharmacological chaperone and promoting the export from the ER. By relieving the misfolded protein load, rod cells can potentially regrow outer segments, preventing further cell death and potentially restoring function. In contrast, in the iCasp9 model, there is direct activation of apoptosis, an active programmed cell death mechanism that avoids eliciting inflammatory responses. Cells that undergo apoptosis are fragmented into membrane-bound 
A
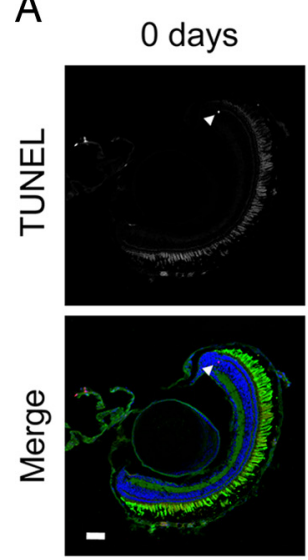

B
2 days
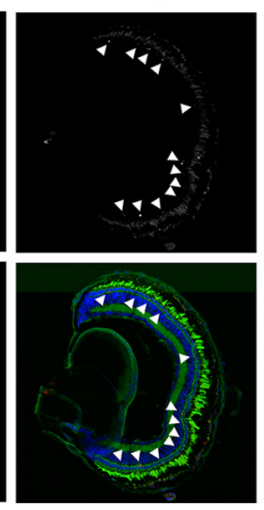

5 days
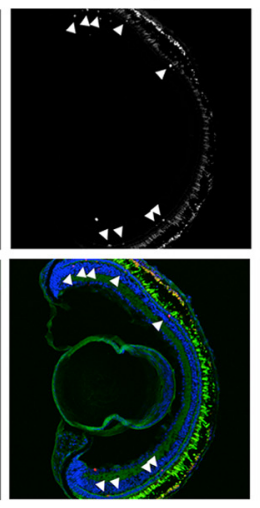

13 days
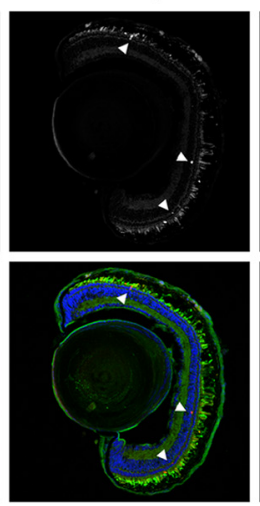

19 days
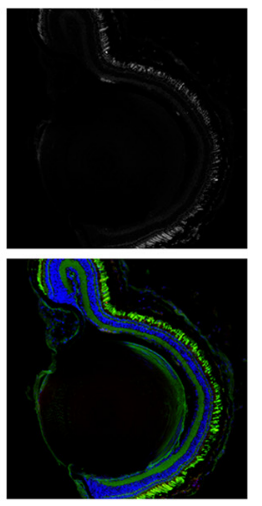

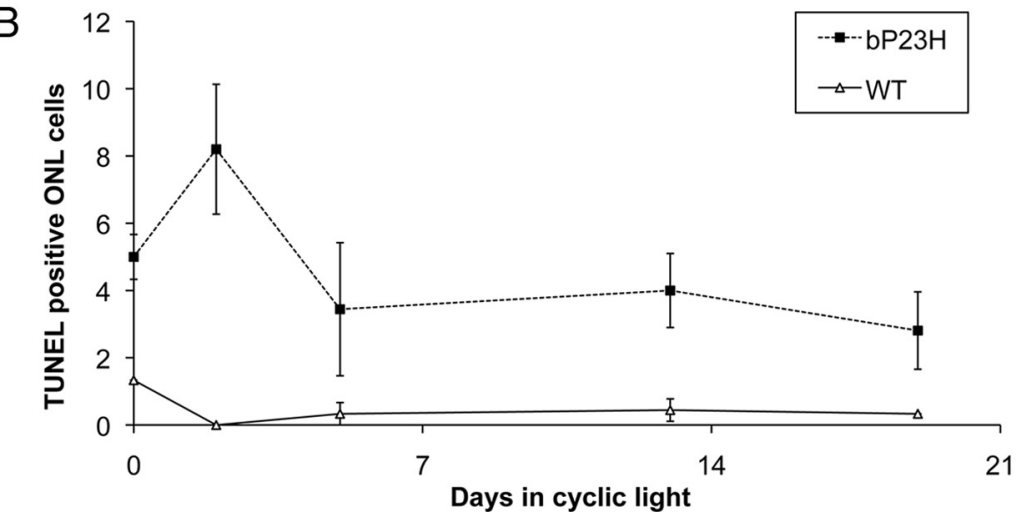

21

Figure 5. Short-term degeneration of bP23H retinas over a 3 week period as monitored by TUNEL. Tadpoles were allowed to develop for $14 \mathrm{~d}$ in complete darkness. At $14 \mathrm{dpf}$, the animals were moved to cyclic light (12 h light/dark cycle) rearing condition. $A$, TUNEL-stained (red) retinal sections of animals at 2, 5, 13, and $19 \mathrm{~d}$ in cyclic light. TUNEL-positive cells in the 0 ONL are indicated by arrowheads. WGA, Green; Hoechst nuclear stain, blue. Scale bar, $100 \mu \mathrm{m}$. $\boldsymbol{B}$, The number of TUNEL-positive cells in the ONL were counted over the 3 week cyclic light period. Graph represents the number of TUNEL-positive ONL cells per cryosection, averaged over at least three samples per time point ( $n>3$, mean \pm SEM). For each sample, TUNEL-positive cells were counted in three sections obtained from the center of the tissue block. WT, Wild type.

apoptotic bodies, which are subsequently phagocytosed and cleared by neighboring cells and/or macrophages. In retinas in which cells are lost as a result of cell death, regeneration of new cells would be required to repopulate the retina; however, we did not observe any such recovery in the iCasp9 model, in contrast to recently published observations (Choi et al., 2011).

The concentration and compartmentalization of glutamate and taurine are indices of metabolic homeostasis in photoreceptors across species, including fish, reptiles, and mammals (Marc et al., 1995, 1998a,b, 2007; Marc and Jones, 2002). Glutamate is necessary for protein synthesis, energetics, and neurotransmission in excitatory neurons (Frigerio et al., 2008). Taurine is widely involved in membrane stabilization, osmoregulation, neuromodulation, regulation of calcium homeostasis, and antioxidation (Bouckenooghe et al., 2006). In this study, we found that there is a high concentration and normal distribution of glutamate and taurine in the soma and inner segments of degenerating $\mathrm{bP} 23 \mathrm{H}$ rods, similar to that found in normal rods and cones, suggesting that they are metabolically active despite hosting degenerated outer segments. Metabolomic profiling suggests that degenerating $\mathrm{bP} 23 \mathrm{H}$ rods remain metabolically active and are indistinguishable from non-degenerating cones using the current panel of small-molecule probes.

Rods that regrow outer segments display diverse smallmolecule profiles based on taurine, glutamate, aspartate, and glutathione levels. During early stages of degeneration, some photoreceptor levels of glutamate, aspartate, and glutathione spike only to become taurine and glutamate deficient before presumed photoreceptor death or recovery (Marc et al., 1998a,b, 2007). We hypothesize that elevated levels of glutamate and aspartate contribute to energy and protein synthesis requirements to elongate soma and outer segments. For example, neurons can alter concentrations of glutamate and aspartate, whose metabolism are intricately linked, to accommodate demands for gluconeogenesis, ureogenesis, and the generation of protein and amino acids (Frigerio et al., 2008). Although elevated glutathione levels are associated with oxidative stress in retinal cells, we further propose that rods elevate glutathione levels to modulate protein levels as pharmacological inhibitors of glutathione synthesis in stressed retinal cells decrease levels of protein synthesis (Castagn é and Clarke, 1998). Less clear is the significance of lower taurine levels in recovering rods given its importance in homeostasis and antioxidation. However, we found that the rods and cones of young/developing X. laevis (stage 47/48) do not display taurine immunoreactivity (data not shown), suggesting that $X$. laevis rods possess alternate metabolic pathways for osmoregulation and antioxidation. Alternative osmolyte systems used by nonmammalians include polyhydric alcohols, such as glycerol and sucrose; free amino acids and amino acid derivatives, such as $\beta$-alanine, and urea and methylamines, such as trimethylamine$\mathrm{N}$-oxide (Yancey, 2005). In the recovering $\mathrm{P} 23 \mathrm{H}$ retina, a decrease in taurine levels might be a requirement for rods to regrow outer segments. Using computational metabolic profiling in degenerating bP23H retina, we demonstrated that dysmorphic de- 


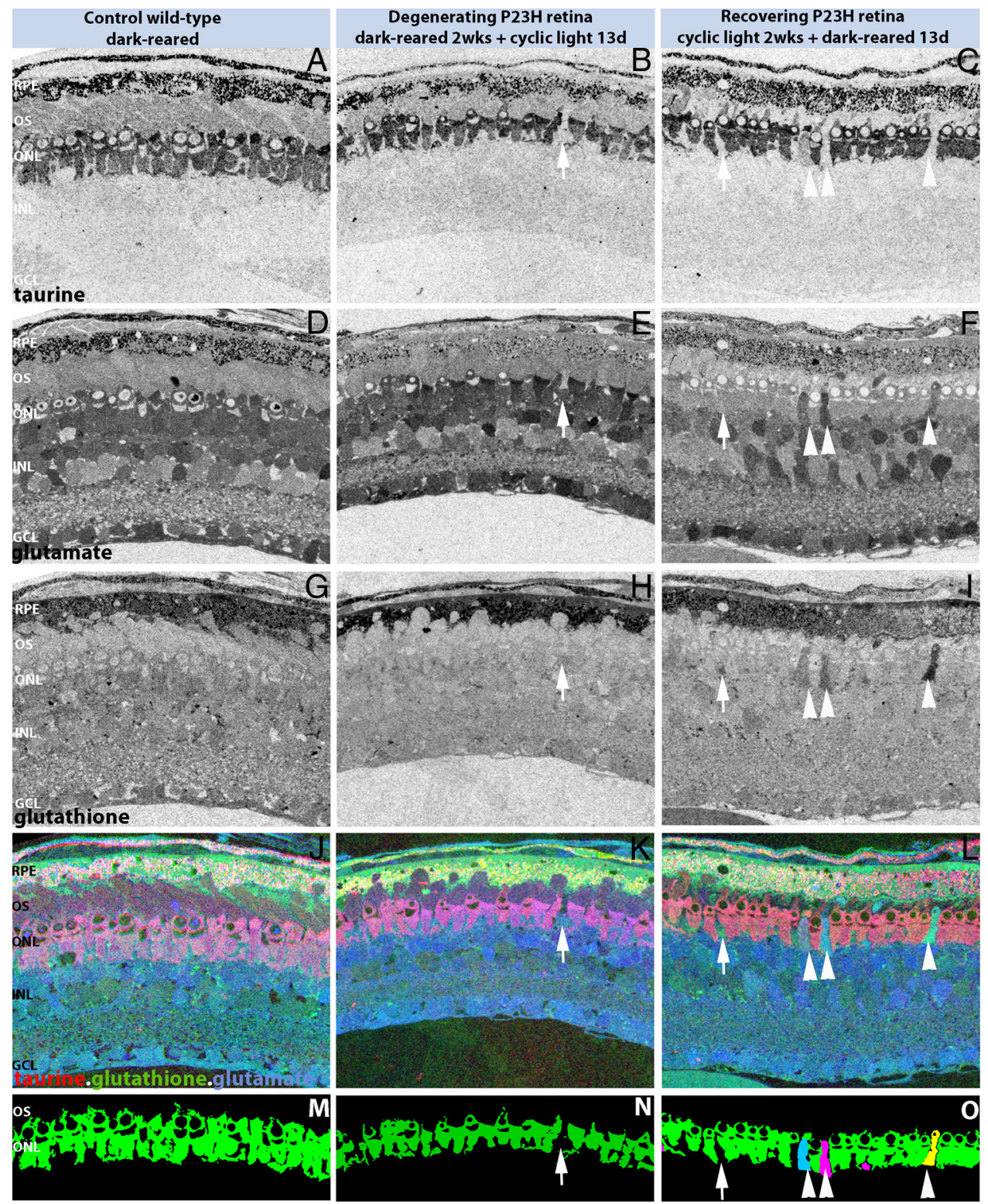

Figure 6. Short-term metabolic changes in bP23H retinas. $A-I$, Visualization of metabolic content and distribution by quantitative metabolite mapping on $90 \mathrm{~nm}$ sections probed with Igs specific to each metabolite and visualized with silver intensification. $\boldsymbol{J}-\boldsymbol{L}$, Red-green-blue channels represent taurine-glutathione-glutamate mapping. The pink and red compartments in the photoreceptor outer segments (OS) and inner segments (IS) contain distinct taurine-glutamate-glutathione mixtures, whereas various blue-to-azure cells in the interneuron layer (INL) and ganglion cell layer (GCL) are neurons with distinctive glutamate content. The green-yellow background of RPE reflect high levels of glutathione. $\mathbf{M}-\mathbf{0}$, Theme maps display results of multispectral analyses and clustering analyses to extract all distinct molecular phenotypes in the photoreceptor layer. Signals from glutamate, glutathione, taurine, aspartate, GABA, glycine, arginine, alanine, and CRALBP were used for multispectral analyses and clustering analyses. $M, N$, Theme maps show that the metabolic profiles of rods and cones in control and degenerating retina were similar; whereas in recovering retina, rods displayed variability in their metabolic profile because of variability in their taurine, glutamate, and glutathione content. Arrows and arrowheads indicate Müller glia and regenerating rods, respectively.

generating rods remain metabolically competent and that regrowth of outer segment is associated with various metabolic states involving decreased levels in taurine and spikes in glutamate, aspartate, and glutathione levels. It will be interesting to determine whether similar effects are observed in other models of RP.

Although we found that dystrophic bP23H rods are metabolically active and able to regenerate outer segments, our observations also indicate that they cannot maintain this state indefinitely as rods are gradually lost. It would be informative to further characterize the cytoprotective mechanisms responsible for this protected state and determine the molecular/metabolic threshold at which $\mathrm{P} 23 \mathrm{H}$ photoreceptors are unable to recover. This information could be potentially used to determine a treatment to buffer this condition or broaden the window of recoverability. Any therapeutic interventions to rescue sick photoreceptors will have to occur in the early phase, before the photoreceptor dies (Marc et al., 2003). The results from this study also suggest that $\mathrm{P} 23 \mathrm{H}$ rhodopsin RP patients may have a pool of rod photoreceptor cells that are dystrophic but still viable, which could be targeted with therapeutic interventions that slow, prevent, or even reverse degeneration. Based on previous examples of restoring function to dysfunctional photoreceptors, such an approach is likely to restore vision (Cideciyan et al., 2008; Busskamp et al., 2010). By comparing metabolic abnormalities observed in our animal models and human RP patients, we may be able to categorize RP and tailor therapeutic interventions to subsets of patients. 


\section{References}

Berson EL (1993) Retinitis pigmentosa. The Friedenwald Lecture. Invest Ophthalmol Vis Sci 34:1659-1676.

Bouckenooghe T, Remacle C, Reusens B (2006) Is taurine a functional nutrient? Curr Opin Clin Nutr Metab Care 9:728-733.

Busskamp V, Duebel J, Balya D, Fradot M, Viney TJ, Siegert S, Groner AC, Cabuy E, Forster V, Seeliger M, Biel M, Humphries P, Paques M, Mohand-Said S, Trono D, Deisseroth K, Sahel JA, Picaud S, Roska B (2010) Genetic reactivation of cone photoreceptors restores visual responses in retinitis pigmentosa. Science 329:413-417.

Castagn é V, Clarke PG (1998) Cooperation between glutathione depletion and protein synthesis inhibition against naturally occurring neuronal death. Neuroscience 86:895-902.

Choi RY, Engbretson GA, Solessio EC, Jones GA, Coughlin A, Aleksic I, Zuber ME (2011) Cone degeneration following rod ablation in a reversible model of retinal degeneration. Invest Ophthalmol Vis Sci 52:364-373.

Cideciyan AV, Aleman TS, Boye SL, Schwartz SB, Kaushal S, Roman AJ, Pang JJ, Sumaroka A, Windsor EA, Wilson JM, Flotte TR, Fishman GA, Heon E, Stone EM, Byrne BJ, Jacobson SG, Hauswirth WW (2008) Human gene therapy for RPE65 isomerase deficiency activates the retinoid cycle of vision but with slow rod kinetics. Proc Natl Acad Sci U S A 105:15112-15117.

Dryja TP, McGee TL, Reichel E, Hahn LB, Cowley GS, Yandell DW, Sandberg MA, Berson EL (1990) A point mutation of the rhodopsin gene in one form of retinitis pigmentosa. Nature 343:364-366.

Frigerio F, Casimir M, Carobbio S, Maechler P (2008) Tissue specificity of mitochondrial glutamate pathways and the control of metabolic homeostasis. Biochim Biophys Acta 1777:965-972.

Galy A, Roux MJ, Sahel JA, Léveillard T, Giangrande A (2005) Rhodopsin maturation defects induce photoreceptor death by apoptosis: a fly model for RhodopsinPro23His human retinitis pigmentosa. Hum Mol Genet 14:2547-2557.

Gorbatyuk MS, Knox T, LaVail MM, Gorbatyuk OS, Noorwez SM, Hauswirth WW, Lin JH, Muzyczka N, Lewin AS (2010) Restoration of visual function in $\mathrm{P} 23 \mathrm{H}$ rhodopsin transgenic rats by gene delivery of BiP/Grp78. Proc Natl Acad Sci U S A 107:5961-5966.

Hamm LM, Tam BM, Moritz OL (2009) Controlled rod cell ablation in transgenic Xenopus laevis. Invest Ophthalmol Vis Sci 50:885-892.

Hartong DT, Berson EL, Dryja TP (2006) Retinitis pigmentosa. Lancet 368:1795-1809.

Jones BW, Watt CB, Frederick JM, Baehr W, Chen CK, Levine EM, Milam AH, Lavail MM, Marc RE (2003) Retinal remodeling triggered by photoreceptor degenerations. J Comp Neurol 464:1-16.

Jozwick C, Valter K, Stone J (2006) Reversal of functional loss in the P23H-3 rat retina by management of ambient light. Exp Eye Res 83:1074-1080.

Kaushal S, Khorana HG (1994) Structure and function in rhodopsin. 7. Point mutations associated with autosomal dominant retinitis pigmentosa. Biochemistry 33:6121-6128.

Lin JH, Walter P, Yen TS (2008) Endoplasmic reticulum stress in disease pathogenesis. Annu Rev Pathol 3:399-425.

Marc RE, Jones BW (2002) Molecular phenotyping of retinal ganglion cells. J Neurosci 22:413-427.

Marc RE, Murry RF, Basinger SF (1995) Pattern recognition of amino acid signatures in retinal neurons. J Neurosci 15:5106-5129.

Marc RE, Murry RF, Fisher SK, Linberg KA, Lewis GP, Kalloniatis M (1998a)
Amino acid signatures in the normal cat retina. Invest Ophthalmol Vis Sci 39:1685-1693.

Marc RE, Murry RF, Fisher SK, Linberg KA, Lewis GP (1998b) Amino acid signatures in the detached cat retina. Invest Ophthalmol Vis Sci 39:1694-1702.

Marc RE, Jones BW, Watt CB, Strettoi E (2003) Neural remodeling in retinal degeneration. Prog Retin Eye Res 22:607-655.

Marc RE, Jones BW, Anderson JR, Kinard K, Marshak DW, Wilson JH, Wensel T, Lucas RJ (2007) Neural reprogramming in retinal degeneration. Invest Ophthalmol Vis Sci 48:3364-3371.

Milam AH, Li ZY, Fariss RN (1998) Histopathology of the human retina in retinitis pigmentosa. Prog Retin Eye Res 17:175-205.

Moritz OL, Tam BM (2010) Recent insights into the mechanisms underlying light-dependent retinal degeneration from $X$. laevis models of retinitis pigmentosa. Adv Exp Med Biol 664:509-515.

Noorwez SM, Kuksa V, Imanishi Y, Zhu L, Filipek S, Palczewski K, Kaushal S (2003) Pharmacological chaperone-mediated in vivo folding and stabilization of the $\mathrm{P} 23 \mathrm{H}$-opsin mutant associated with autosomal dominant retinitis pigmentosa. J Biol Chem 278:14442-14450.

Noorwez SM, Malhotra R, McDowell JH, Smith KA, Krebs MP, Kaushal S (2004) Retinoids assist the cellular folding of the autosomal dominant retinitis pigmentosa opsin mutant $\mathrm{P} 23 \mathrm{H}$. J Biol Chem 279:16278-16284.

Saliba RS, Munro PM, Luthert PJ, Cheetham ME (2002) The cellular fate of mutant rhodopsin: quality control, degradation and aggresome formation. J Cell Sci 115:2907-2918.

Shintani K, Shechtman DL, Gurwood AS (2009) Review and update: current treatment trends for patients with retinitis pigmentosa. Optometry 80:384-401.

Sohocki MM, Daiger SP, Bowne SJ, Rodriquez JA, Northrup H, Heckenlively JR, Birch DG, Mintz-Hittner H, Ruiz RS, Lewis RA, Saperstein DA, Sullivan LS (2001) Prevalence of mutations causing retinitis pigmentosa and other inherited retinopathies. Hum Mutat 17:42-51.

Tam BM, Moritz OL (2006) Characterization of rhodopsin P23H-induced retinal degeneration in a Xenopus laevis model of retinitis pigmentosa. Invest Ophthalmol Vis Sci 47:3234-3241.

Tam BM, Moritz OL (2007) Dark rearing rescues P23H rhodopsin-induced retinal degeneration in a transgenic Xenopus laevis model of retinitis pigmentosa: a chromophore-dependent mechanism characterized by production of N-terminally truncated mutant rhodopsin. J Neurosci 27:9043-9053.

Tam BM, Moritz OL, Hurd LB, Papermaster DS (2000) Identification of an outer segment targeting signal in the $\mathrm{COOH}$ terminus of rhodopsin using transgenic Xenopus laevis. J Cell Biol 151:1369-1380.

Tam BM, Qazalbash A, Lee HC, Moritz OL (2010) The dependence of retinal degeneration caused by the rhodopsin $\mathrm{P} 23 \mathrm{H}$ mutation on light exposure and vitamin a deprivation. Invest Ophthalmol Vis Sci 51:1327-1334.

Valter K, Kirk DK, Stone J (2008) The potential of ambient light restriction to restore function to the degenerating $\mathrm{P} 23 \mathrm{H}-3$ rat retina. Adv Exp Med Biol 613:193-199.

Wang G, Yang ZQ, Zhang K (2010) Endoplasmic reticulum stress response in cancer: molecular mechanism and therapeutic potential. Am J Transl Res 2:65-74.

Yancey PH (2005) Organic osmolytes as compatible, metabolic and counteracting cytoprotectants in high osmolarity and other stresses. J Exp Biol 208:2819-2830. 